\title{
Comparative Study on Charge Pump Circuits for Harvesting Solar Energy
}

\author{
JeaSang Song, Sang-Don Byeon, SangHak Shin, Son Ngoc \\ Truong, Hyun-Sun Mo, Kyeong-Sik Min ${ }^{\mathrm{a}}$ \\ School of Electrical Engineering, Kookmin University, Seoul 136-702, Korea \\ aemail:mks@kookmin.ac.kr
}

Keywords: Energy harvesting, solar energy, sunlight, charge pump circuits

\begin{abstract}
Sunlight is one of useful energy harvesting sources that can be found everywhere. In spite of this ubiquitous availability, sunlight intensity is dynamically changing. Thus Maximum Power Point Tracking (MPPT) circuits are needed to harvest the maximum available energy from the solar cell, regardless of sunlight intensity variation. In this paper, three charge pump circuits for solar energy harvesting are discussed in terms of the output current and energy efficiency. The first charge pump can change its pumping frequency to track the maximum power point at a given sunlight condition. The second circuit can reconfigure its architecture according to the sunlight variation to maximize its output current with varying the sunlight intensity. In the third pump circuit, the reconfigurable architecture can be combined with the variable pumping frequency to keep the power efficiency high at any sunlight condition.
\end{abstract}

\section{Introduction}

Sunlight is very useful energy source that can be converted to electrical energy very easily using simple power conversion circuits. Solar energy can be found everywhere and its density can be high when sunlight is strong. Using solar cells, DC voltage and DC current can be directly obtained from sunlight not being converted from AC power. Compared with the other energy sources that can be harvested from the environment such as vibration, wind, wave, temperature gradient, etc, sunlight has the following advantages; ubiquitous availability of energy source, wide dynamic range of energy density, and simplicity of energy conversion systems that can produce DC current and DC voltage directly in solar harvesting.

The power conversion systems that can obtain electrical energy from environmental sunlight can be categorized into two; the one is the inductor-based power conversion circuits that are more suitable to extract a large amount of energy from sunlight and the other is the capacitor-based power conversion circuits that use on-chip pumping capacitors for small-scale energy harvesting. The capacitor-based power conversion circuits can be implemented without using inductors that should be attached externally to the chip. By doing so, they can be considered more suitable to Wireless Sensor Networks (WSNs) that need very small amount of energy for their operation. Also, compared to the inductor-based power conversion circuits, the capacitor-based harvesting circuits can be made with lower cost due to the simpler architecture.

We focus on the capacitor-based power conversion circuits because we target to develop simple and low-cost solar harvesting circuits, in this paper. One obvious problem in converting sunlight to electrical energy is that sunlight is not static but dynamically changing. As a result of this dynamic changing of sunlight, an amount of solar energy that can be converted to electrical energy can be changed according to sunlight intensity variation, too. When sunlight is very strong, the amount of solar energy that can be harvested can be large. On the contrary, at weak sunlight, the amount of solar energy becomes small thereby the converted amounts of electrical energy become small, too. Figures 1(a) and (b) show the current-voltage and power-voltage relationships of solar cell for various sunlight conditions, respectively [1]. In Figures 1(a) and (b), the Maximum Power Point (MPP) means the bias point of delivering the maximum amount of the solar cell's power at a given sunlight intensity. Here $\mathrm{V}_{\mathrm{SC}}$ and $\mathrm{I}_{\mathrm{SC}}$ are the solar cell's voltage and current, respectively. 


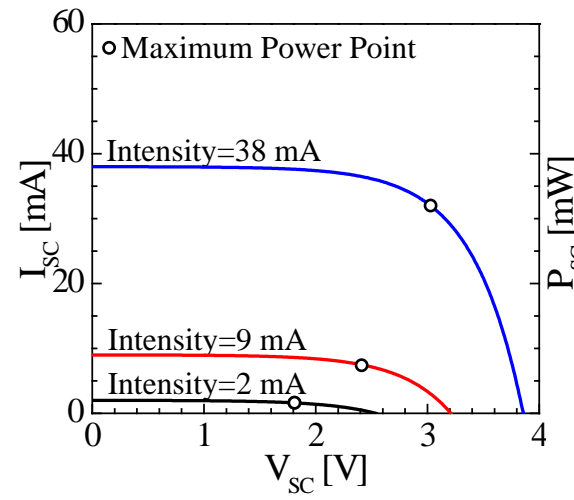

(a)

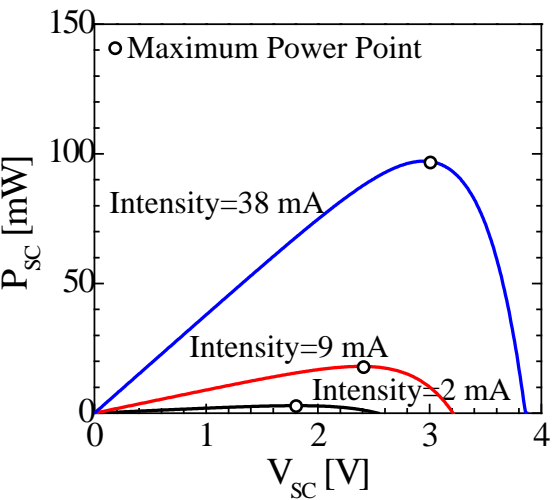

(b)

Fig. 1. (a) The relationship of solar cell's current $\left(\mathrm{I}_{\mathrm{SC}}\right)$ and voltage $\left(\mathrm{V}_{\mathrm{SC}}\right)$ according to sunlight intensity variation [1] (b) The relationship of solar cell's power $\left(\mathrm{P}_{\mathrm{SC}}\right)$ and voltage $\left(\mathrm{V}_{\mathrm{SC}}\right)$ according to sunlight intensity variation [1]

\section{Three charge pumps circuits for harvesting solar energy}

As mentioned earlier, the MPPs can be changed according to the variation of sunlight intensity. To maximize the power efficiency of solar harvesting circuits at a given sunlight condition, we need to add the Maximum Power Point Tracking (MPPT) circuit to the power conversion circuit. From the previous publications, we could find three MPPT circuits. Figure 2 shows the conceptual schematic of the first change pump circuit which can change its pumping frequency to track the MPPs [2]. Three sunlight conditions are shown in Figure 2, which are the weak, moderate, and strong sunlight conditions, respectively. At weak sunlight, the pumping frequency is slow to deliver a small amount of output current to the load. At strong sunlight, the pumping frequency becomes faster thus the charge pump can deliver a larger amount of current to the load.
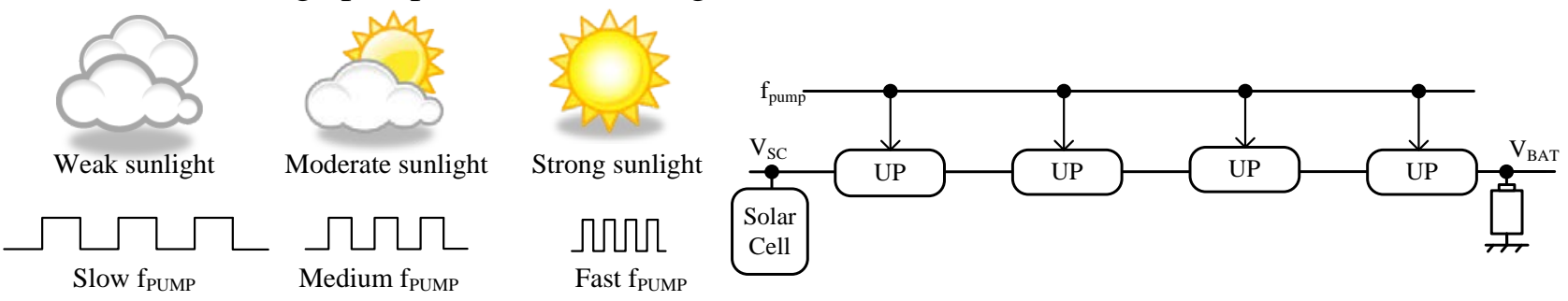

Fig. 2. The conceptual schematic of the first charge pump which can change its pumping frequency according to the sunlight variation [2]

Instead of changing the pumping frequency according to the sunlight variation, we can change the charge pump architecture which can be made reconfigurable, for tracking MPPs, as shown in Figures 3(a), (b), and (c) [3]. Figure 3(a) shows the serial architecture. In Figure 3(a), the smaller amount of pumping current can be delivered, but the boosting ratio can be higher than Figures 3(b) and (c). The serial architecture in Figure 3(a) is very suitable to the weak sunlight condition, because the low $\mathrm{V}_{\mathrm{OC}}$ (open-ckt voltage) at weak sunlight needs the high boosting ratio and the low I $_{\text {SC }}$ (short-ckt current) can produce only the small amount of output current. Figure 3(c) represents the parallel architecture that can deliver the larger amount of pumping current with the lower boosting ratio than Figures 3(a) and (b). The parallel architecture is very suitable to the strong sunlight. Here, the high $\mathrm{V}_{\mathrm{OC}}$ at the strong sunlight intensity needs the lower boosting ratio and the high $\mathrm{I}_{\mathrm{SC}}$ can deliver the larger output current than Figures 3(a) and (b).

Combining the reconfigurable architecture with the variable pumping frequency can improve the power efficiency and output current better than the first circuit in Figure 2 and second circuit in Figure 3 [1]. Figures 4(a), (b), and (c) show the conceptual schematics of serial, parallel-serial, and parallel architecture which are combined with the variable pumping frequency, respectively [1]. 


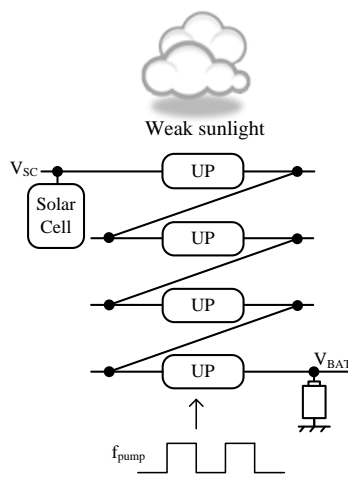

(a)

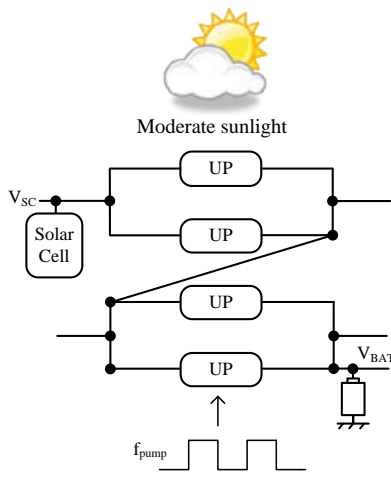

(b)

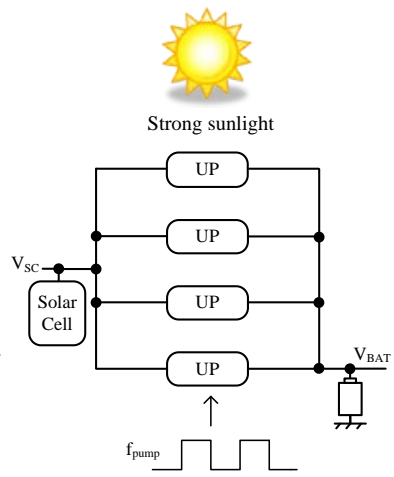

(c)

Fig. 3. The conceptual schematic of the second charge pump which can reconfigure its architecture according to the sunlight variation [3]. (a) The weak sunlight condition, (b) The moderate sunlight condition, and (c) The strong sunlight condition

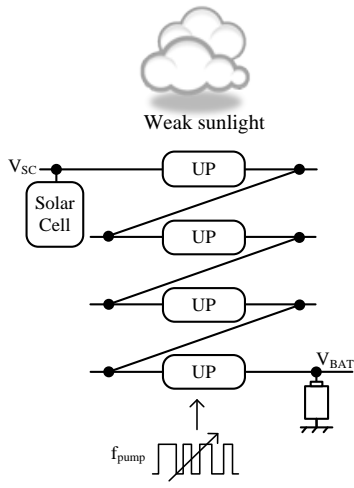

(a)

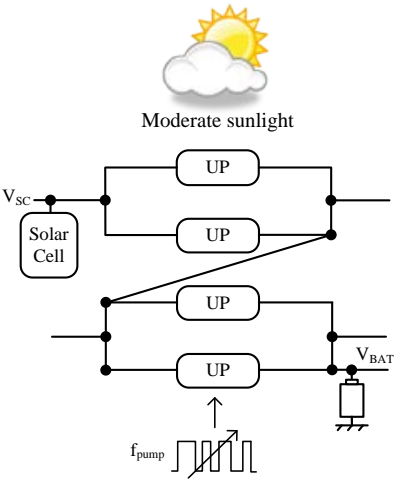

(b)

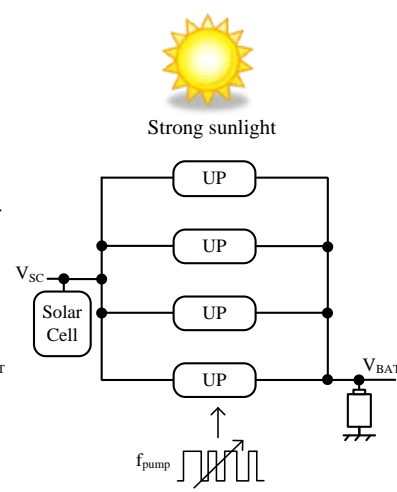

(c)

Fig. 4. The conceptual schematic of the third charge pump which can change both its architecture and pumping frequency according to the sunlight variation [1]. (a) The weak sunlight condition, (b) The moderate sunlight condition, and (c) The strong sunlight condition

\section{Comparison of three charge pump circuits and discussion}

First of all, we compared the first circuit in Figure 2 with the variable pumping frequency [2] and the reconfigurable charge pump circuit in Figure 3 [3]. In terms of the dynamic range of output current, the circuit simulation indicates that the reconfigurable circuit can change the output current more than the variable pumping frequency [2]. [3]. However, in terms of accuracy of MPPT, the variable pumping frequency is better than the reconfigurable charge pump. This is because the pumping frequency can be changed continuously in Figure 2 [2]. In contrast, the reconfigurable pump can decide its architecture among only three that are serial, parallel-serial, and parallel, as indicated in Figures 3(a), (b), and (c) [3].

In Figure 5(a), the second circuit with 3 reconfigurable architectures in Figure 3 is compared with the third circuit which can change its both pumping frequency and architecture, as shown in Figure 4. When we fixed the pumping frequency around $7 \mathrm{MHz}$ and changed only the architecture, the percentage output current of the second charge pump is only $70 \%$ of the maximum output current, in average of 3 sunlight conditions [1]. Similarly, when we fixed the pumping frequency around $15 \mathrm{MHz}$ and changed only the architecture according to the sunlight variation, the percentage output current of the second circuit is less than $70 \%$ of the maximum available output current [1]. When we changed both the pumping frequency and architecture according to the sunlight variation, as shown in Figure 4, the percentage output current is as much as $92 \%$ of the maximum available output current [1]. This comparison between the second charge pump and the third circuit indicates that the third circuit can deliver the output current by $20 \%$ more than the second circuit, as we see in Figure 5(a). Figure 5(b) compares the output current of the third circuit with respect to MPPs when the sunlight intensity is varied from $1.5 \mathrm{~mA}$ to $58 \mathrm{~mA}$ [1]. For this wide 
range of sunlight intensity, the third circuit seems that it can deliver almost the same amounts of output current with the maximum available amounts that can be obtained from the solar cell [1].

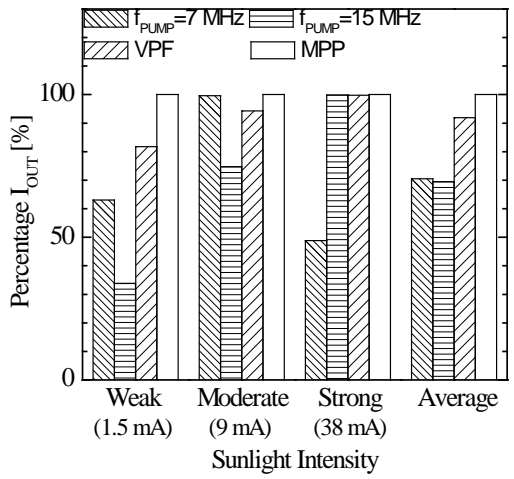

(a)

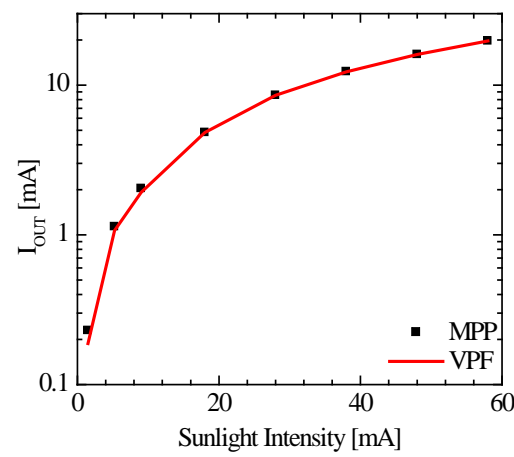

(b)

Fig. 5. (a) The percentage $\mathrm{I}_{\mathrm{OUT}}$ for various sunlight conditions [1]. The second circuit with the pumping frequencies of $7 \mathrm{MHz}$ and $15 \mathrm{MHz}$ is compared with the third pump circuit which is represented by 'VPF'. Here MPP means the maximum output currents that can be obtained from the solar cell. (b) The output current comparison between the simulated maximum output currents (MPP) and the third pump circuit in Figure 4 [1].

\section{Conclusion}

Sunlight is one of useful energy harvesting sources that can be found everywhere. In spite of this ubiquitous availability, sunlight intensity is dynamically changing thus Maximum Power Point Tracking (MPPT) circuits are needed to harvest the maximum available energy from the solar cell, regardless of sunlight intensity variation. In this paper, three charge pump circuits with MPPT function were discussed in terms of the output current and energy efficiency. The first charge pump could change its pumping frequency to track the maximum power point at a given sunlight condition. The second circuit could reconfigure its architecture to maximize its output current with varying the sunlight intensity. In the third pump circuit, the reconfigurable architecture could be combined with the variable pumping frequency to keep the power efficiency high at any sunlight condition.

\section{Acknowledgement}

The work was financially supported by NRF-2011-220-D00089, NRF-2011-0030228, NRF-2013K1A3A1A25038533, NRF-2013R1A1A2A10064812, and BK Plus with the Educational Research Team for Creative Engineers on Material-Device-Circuit Co-Design (Grant No: 22A20130000042), funded by the National Research Foundation of Korea (NRF), and by Global Scholarship Program for Foreign Graduate Students at Kookmin Univ. The CAD tools were supported by IC Design Education Center (IDEC), Daejeon, Korea..

\section{References}

[1] Jeong-Heon Kim, Sang-Don Byeon, Hyun-Sun Mo, Kyeong-Sik Min. Reconfigurable charge pump circuit with variable pumping frequency scheme for harvesting solar energy under various sunlight intensities [J]. International Journal of Photoenergy, 2014 (437641), 1-9.

[2] H. Shao, C. Y. Tsui, W. H. Ki. The design of a micro power management system for applications using photovoltaic cells with the maximum output power congrol [J]. IEEE Trans. VLSI systems, 200917 (8) 1138-1142.

[3] E. S. Lee, J. M. Choi, Y. S. Kim, S. J. Ham, J. H. Kim, S. D. Byeon, K. S. Min. Sunlight-variation-adaptive charge pump circuit with self-reconfiguration for small-scale solar energy harvesting [J]. IEICE Electronics Express, 20129 (17) 1423-1433. 